\title{
Sleep quality and emergence delirium in children undergoing strabismus surgery: a comparison between preschool- and school- age patients
}


Hyeon Jeong Lee ${ }^{1,2}$ (C) and Jeong-Min Hong ${ }^{1,2^{*}}$ (])

\begin{abstract}
Background: Emergence delirium (ED) is common in pediatric patients undergoing general anesthesia with sevoflurane. Preoperative sleep quality is associated with the risk factors for ED. However, research on the relationship between sleep quality and ED is limited. We aimed to investigate the relationship between ED and preoperative sleep quality in pediatric patients undergoing strabismus surgery.
\end{abstract}

Methods: This clinical trial included pediatric patients aged 4-12 years who underwent elective strabismus surgery. The patients and their parents were questioned about the patients' preoperative sleep quality using the Pittsburgh Sleep Quality Index (PSQI) questionnaire. For anesthesia induction, thiopental $(5 \mathrm{mg} / \mathrm{kg})$ and rocuronium $(0.6 \mathrm{mg} / \mathrm{kg})$ were used, and anesthesia was maintained with sevoflurane (minimum alveolar concentration, 1-1.5). After administration of a reversal drug, extubation was performed, and the patients were transferred to a post-anesthesia recovery unit. At 10 min after extubation, the degree of ED was measured using the pediatric anesthesia emergence delirium (PAED) and Watcha scale scores.

Results: Of the 62 enrolled patients, three pediatric patients were excluded. The overall incidence of ED was 22\%. A total of 59 patients were divided into the two groups. The ED group and the non-ED group comprised 13 and 46 patients. Age, height and weight were significantly lower in the ED group than in the non-ED group. Preoperative PSQI and Watcha scale score were significantly higher in the ED group than in the non-ED group. Multivariate analysis showed that age (adjusted OR [95\% Cl]: 0.490 [0.290-0.828], $p=0.008$ ) and preoperative PSQI score (adjusted OR [95\% Cl]: 2.149[1.224-3.771], $p=0.008$ ) was associated with ED. In sub-group analysis, PAED scale and Watcha scale scores showed a moderate correlation with preoperative sleep quality in preschool-age patients.

Conclusion: In conclusion, the incidence of ED tended to be higher in younger age and poorer preoperative sleep quality in pediatric patients. In particular, the poorer sleep quality score was associated with higher incidence of ED in the preschool-age. Large-scale clinical studies and long-term follow-up studies on ED and sleep quality are required.

Trial registration: This study was registered with Clinicaltrials.gov (NCT03332407) at November 5th 2017.

\footnotetext{
*Correspondence: ccarrot@pusan.ac.kr

2 Department of Anesthesia and Pain Medicine, School of Medicine,

Pusan National University, 1-10, Ami-dong, Seo-gu, Busan 49241, Republic of Korea

Full list of author information is available at the end of the article
} original author(s) and the source, provide a link to the Creative Commons licence, and indicate if changes were made. The images or other third party material in this article are included in the article's Creative Commons licence, unless indicated otherwise in a credit line to the material. If material is not included in the article's Creative Commons licence and your intended use is not permitted by statutory regulation or exceeds the permitted use, you will need to obtain permission directly from the copyright holder. To view a copy of this licence, visit http://creativecommons.org/licenses/by/4.0/. The Creative Commons Public Domain Dedication waiver (http://creativeco mmons.org/publicdomain/zero/1.0/) applies to the data made available in this article, unless otherwise stated in a credit line to the data. 
Keywords: Emergence delirium, Preoperative sleep quality, Pediatrics, Strabismus surgery

\section{Background}

Emergence delirium (ED) is defined as confusing mentality, irritability, inconsolable crying, and disorientation at the time of emergence from anesthesia [1]. To date, its physiological mechanism has not yet been fully elucidated. However, some possible risk factors for ED, such as preschool age, rapid emergence from inhalational anesthesia, presence of pain, stressful induction, noisy and unfriendly environment, duration of anesthesia, premedication, anxiety, anesthetic techniques, and patient's personality, have been identified [1, 2]. According to previous reports, the incidence of ED varies from 0.25 to $95 \%$, and it frequently occurs in pediatric patients. Fortunately, ED is a self-limiting phenomenon that lasts for approximately 5 to $15 \mathrm{~min}$ [3]. However, it has been reported to frequently cause catheter removal, attempts at self-extubation, and injuries to patients and staff [4]. In addition, children who experience ED during the postanesthesia period can be exposed to a risk of falling from the bed or aggravation of wounds. Several studies have investigated the prevention of ED with drugs such as propofol, midazolam, ketamine and dexmedetomidine, given at the end of surgery. However, superiority over effective methods has not been defined [5-8].

Sleep is an essential factor in maintaining the physiological functions of the human body. Sleep is crucial for our daily living and related to the prognosis of patients treated under general anesthesia. If a person has a poor sleep quality preoperatively, it has been reported as a risk factor for severe postoperative pain [9-11]. Previous studies have reported that preoperative sleep quality is associated with postoperative delirium [12-15]. Postoperative pain, personality disorder, maladaptive behavior, anxiety, and postoperative delirium are associated with sleep quality and are also risk factors for the occurrence of ED [16-20]. However, no study has demonstrated the relationship between sleep quality and ED in pediatric patients. In particular, there have been no studies on differences in this relationship according to the age of children. We aimed to investigate the relationship between ED and preoperative sleep quality in pediatric patients undergoing strabismus surgery.

\section{Materials and methods Study design}

The Institutional Review Board of Pusan National University Hospital, Busan, Korea, approved this study (1704-001-067). The investigators obtained informed consent from both patients and parents on the day of the pre-anesthetic evaluation visit.

The study protocol included patients of 4 to 12 years of age who underwent elective strabismus surgery. The components of exclusion criteria are children with disorders or taking medications known to affect blood pressure, sleep, breathing, and neuro-cognitive or behavioral functioning.

The investigators questioned the patients and their parents about their preoperative daily sleep quality using the Pittsburgh Sleep Quality Index (PSQI) questionnaire. The PSQI is a famously used measure of sleep-related problems and sleep quality over the past month [18, 21, 22]. The 19 items of the PSQI are grouped into seven components, scores of which reflect the frequency and severity of sleep problems, including subjective appreciation of sleep quality, sleep latency, sleep duration, habitual sleep efficiency, presence of sleep-disturbing factors, use of sleep medication, and daytime dysfunction. Each component is scored from 0 (no difficulty) to 3 (severe difficulty). All component scores are summed to provide an overall score ranging from 0 to 21 , with a lower score indicating healthier sleep quality. A total score of 5 or greater is used to distinguish "poor" from "good" sleepers.

Before surgery, the patients were not pre-medicated. The parents accompanied their children in the operating room. Anesthesia was induced using thiopental $(5 \mathrm{mg} / \mathrm{kg})$ and rocuronium $(0.6 \mathrm{mg} / \mathrm{kg})$. Anesthesia was maintained with sevoflurane (minimum alveolar concentration, 1-1.5). Investigators performed routine monitoring for all patients, including pulse-oximetry, non-invasive blood pressure assessment, electrocardiography, and endtidal $\mathrm{CO}_{2}$ monitoring. At the end of the surgery, reversal agents (glycopyrrolate $[0.01 \mathrm{mg} / \mathrm{kg}$ and $0.25 \mathrm{mg} / \mathrm{kg}]$ and ketorolac $[0.5-1.0 \mathrm{mg} / \mathrm{kg}]$ ) were administered. The endotracheal tube was removed after recovery of cough and gag reflex and ability to grimace and perform purposeful movements. After extubation, the patients were transported to a post-anesthesia recovery unit (PACU).

As soon as the patients arrived at the PACU, the parents were called and asked to stand by the patients' bedside. At $10 \mathrm{~min}$ after extubation, the degree of ED was measured using pediatric anesthesia emergence delirium (PAED) and Watcha scale scores. The PAED scale as shown in supplemental Table 1, has five items scored from 0 to 4 (with reverse scoring where applicable). The scores were summed to obtain a total score ranging from 
0 to 20 . A total score of 10 or greater was used to describe ED.

Meanwhile, the Watcha scale is a four-point scale, as shown in supplemental Table 2, and a score of 3 or 4 at any time indicates ED. The patient stayed in the PACU for 20 to $50 \mathrm{~min}$, with vital sign monitoring including pulse-oximetry, non-invasive blood pressure assessment, and electrocardiography. The patients were discharged from the PACU based on a modified Aldrete score and the modified post-anesthetic discharge score.

\section{Statistical analysis}

Statisticians performed all analyses using $\mathrm{R}$ (version 4.0.1) and they performed two-tailed tests at a significance level of $5 \%$. The categorical variables were expressed as frequencies and percentages. The normal distribution of the data was tested using KolmogorovSmirnov test. The nonparametric data were presented as medians with interquartile ranges and parametric data as means \pm standard deviations. The results of logistic regression analyses were presented as odds ratio (OR) or adjusted OR with $95 \%$ confidence interval $(95 \% \mathrm{CI})$. The occurrence of ED was judged based on PAED scale score of 10 or greater, and patient data were analyzed by dividing them into ED and non-ED groups. Statisticians performed independent $\mathrm{t}$-tests or Wilcoxon rank-sum tests on continuous variables. They also performed the chi-square test or Fisher's test on categorical variables. Univariate logistic regression analysis was performed to evaluate the association between predictor variables and the occurrence of ED. The independent predictor variables include age, gender, height, weight, body mass index (BMI), anesthesia time, preoperative PSQI score, and ketorolac administered per kilogram. The candidate predictors enrolled in the multivariate logistic regression were those with $p$-values less than 0.05 in the univariate analysis. We performed subgroup analysis to elucidate the correlation between preoperative PSQI score and a PAED scale or a Watcha scale score. We classified the patients into two groups: preschool- and school-age groups. The preschool-age group included patients aged $<7$ years, while the school-age group included patients aged $\geq 7$ years. The correlation between the PAED scale scores and other factors was analyzed using a Spearman correlation analysis. Statistical significance was set at $p<0.05$.

\section{Results}

Of the 62 enrolled patients, three pediatric patients were excluded because of a lack of data and failure to complete the PSQI interview. The study flowchart is displayed in Fig. 1.

The occurrence of ED was determined based on the PAED score, and the demographic data of the patients are presented in Table 1 . The overall incidence of ED was $22 \%$. A total of 59 patients were divided into the two groups. The ED group consisted 13 patients whereas, the non-ED group consisted only 46 patients. There were no significant differences between the two groups in gender, BMI, anesthesia time and amount of ketorolac administered per kilogram of body weight $(p>0.05)$. Age $(p=0.003)$, height $(p=0.008)$ and weight $(p=0.023)$ were significantly lower in the ED group than in the non-ED group. Preoperative PSQI $(p=0.028)$ and Watcha scale score $(p<0.001)$ were significantly higher in the ED group than in the non-ED group. The occurrence of ED was

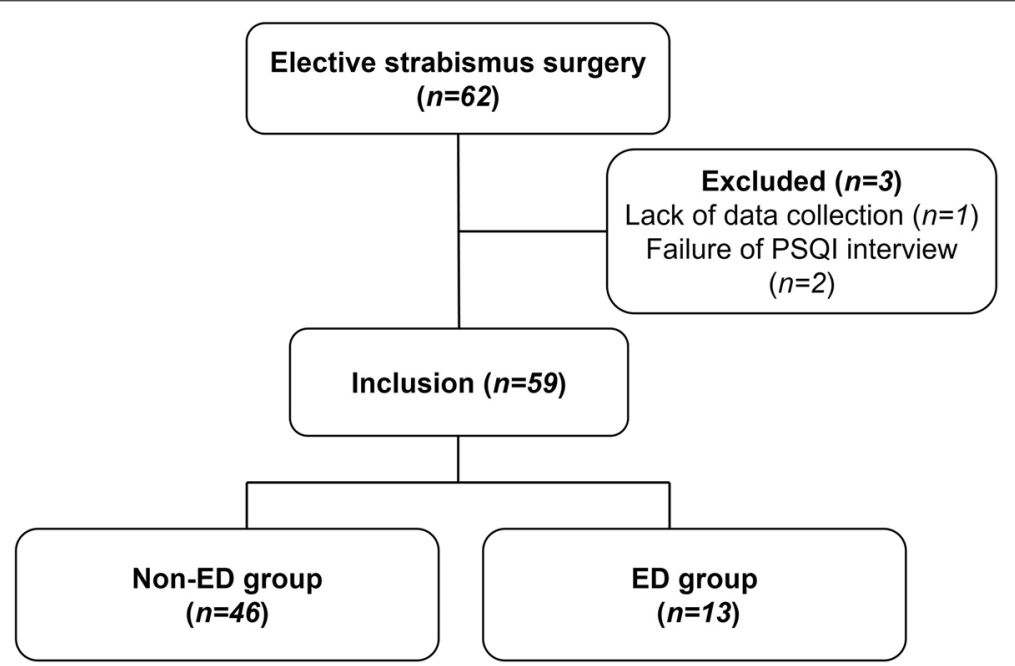

Fig. 1 Study flow chart. PSQI Pittsburg Sleep Quality Index, ED Emergence delirium 
Table 1 Patient demographics

\begin{tabular}{|c|c|c|c|c|}
\hline & \multirow[t]{2}{*}{ Overall $(n=59)$} & \multicolumn{2}{|l|}{ Group } & \multirow[t]{2}{*}{$P$-value } \\
\hline & & $\operatorname{ED}(n=13)$ & Non-ED $(n=46)$ & \\
\hline Age(y) & $6.71 \pm 1.94$ & $5.54 \pm 1.33$ & $7.04 \pm 1.97$ & 0.003 \\
\hline Pre-School age & $29(49.2)$ & $11(84.6)$ & $18(39.1)$ & 0.005 \\
\hline School age & $30(50.8)$ & $2(15.4)$ & $28(60.9)$ & \\
\hline \multicolumn{5}{|l|}{ Gender } \\
\hline Female & $31(52.5)$ & $6(46.2)$ & $25(54.3)$ & 0.835 \\
\hline Male & $28(47.5)$ & $7(53.8)$ & $21(45.7)$ & \\
\hline Height $(\mathrm{cm})$ & $122.51 \pm 14.00$ & $114.94 \pm 9.72$ & $124.65 \pm 14.36$ & 0.008 \\
\hline Weight (Kg) & $26.86 \pm 10.17$ & $22.86 \pm 5.19$ & $27.98 \pm 10.97$ & 0.023 \\
\hline BMI $\left(\mathrm{kg} / \mathrm{m}^{2}\right)$ & $16.64[15.51,18.37]$ & $16.74[16.24,18.34]$ & $16.49[15.27,18.38]$ & 0.499 \\
\hline Anesthesia time (min) & $45.00[37.50,45.00]$ & $45.00[45.00,60.00]$ & $45.00[30.00,45.00]$ & 0.327 \\
\hline Pre-PSQI & $1.41 \pm 1.65$ & $2.38 \pm 1.71$ & $1.13 \pm 1.54$ & 0.028 \\
\hline Ketorolac (mg/kg) & $0.63 \pm 0.19$ & $0.67 \pm 0.16$ & $0.62 \pm 0.20$ & 0.378 \\
\hline Watcha scale & $2.54 \pm 0.750$ & $3.38 \pm 0.650$ & $2.30 \pm 0.591$ & $<0.001$ \\
\hline
\end{tabular}

The incidence of emergence delirium defined on the basis of a PAED scale score of 10 or higher. Data are presented as numbers (\%) or means \pm standard deviations or medians [interquartile ranges]

$B M I$ Body mass index, ED Emergence delirium, Pre-PSQI Preoperative-Pittsburg Sleep Quality Index

11 of $29(37.9 \%)$ in the preschool-age group and 2 of 30 $(6.7 \%)$ in the school-age group. There was a statistically significant association between the occurrence of ED and age-group $(p=0.005)$.

Univariate analysis presented eight predictors which were expected to be associated with the occurrence of ED (Table 2). In univariate analysis, age (OR [95\% CI]: 0.605 [0.399-0.919], $p=0.018$ ), height (OR[95\% CI]: 0.943 [0.894-0.995, $p=0.031])$ and Preoperative PSQI score (OR[95\% CI]: 1.554[1.069-2.259], $p=0.021$ ) were statistically significant. Age, height and preoperative PSQI score were entered in the multivariate logistic regression analysis. The results of variable selection using the backward elimination method were age (adjusted OR [95\% CI]: 0.490 [0.290-0.828], $p=0.008)$ and preoperative
PSQI score (adjusted OR [95\% CI]: 2.149 [1.224-3.771], $p=0.008)$. However, when the occurrence of ED was judged with a Watcha scale score of 3 or higher, preoperative PSQI score (adjusted OR [95\% CI]: 1.077 [0.6751.718], $p=0.756$ ) showed no statistically significant results in multivariate analysis.

The correlations of the PAED and Watcha scale scores with the preoperative PSQI were analyzed via a Spearman correlation analysis in the preschool- and school age group (Table 3). In the preschool-age group, there was a significant correlation between the PAED scale scores and preoperative PSQI (correlation coefficient $=0.377$, $p=0.044)$. There was also a significant correlation between the Watcha scale score and preoperative PSQI (correlation coefficient $=0.376, P=0.044$ ). However, in

Table 2 Univariate and Multivariate analysis of variables related with emergence delirium

\begin{tabular}{|c|c|c|c|c|}
\hline & \multicolumn{2}{|l|}{ Univariate analysis } & \multicolumn{2}{|l|}{ Multivariate analysis } \\
\hline & OR $(95 \% \mathrm{Cl})$ & $P$-value & Adjusted OR $(95 \% \mathrm{Cl})$ & $P$-value \\
\hline Age & 0.605 (0.399-0.919) & 0.018 & $0.490(0.290-0.828)$ & 0.008 \\
\hline Gender & $1.389(0.404-4.776)$ & 0.602 & & \\
\hline Height & $0.943(0.894-0.995)$ & 0.031 & $1.728(0.753-3.967)$ & 0.197 \\
\hline Weight & $0.926(0.842-1.017)$ & 0.108 & & \\
\hline BMI & 0.965 (0.778-1.196) & 0.744 & & \\
\hline Anesthesia time & $1.009(0.977-1.042)$ & 0.598 & & \\
\hline Pre-PSQI & $1.554(1.069-2.259)$ & 0.021 & $2.149(1.224-3.771)$ & 0.008 \\
\hline Ketolorac & $3.994(0.126-126.477)$ & 0.432 & & \\
\hline
\end{tabular}


Table 3 Correlation coefficient between the preoperative PSQI and PAED and Watcha scale scores

\begin{tabular}{|c|c|c|c|}
\hline & & \multicolumn{2}{|c|}{ Preoperative PSQI } \\
\hline & & $\begin{array}{l}\text { Preschool age } \\
(n=29)\end{array}$ & $\begin{array}{l}\text { School age } \\
(n=30)\end{array}$ \\
\hline \multirow[t]{2}{*}{ PAED scale score } & $\begin{array}{l}\text { Correlation coef- } \\
\text { ficient }\end{array}$ & 0.377 & -0.010 \\
\hline & $P$ value & 0.044 & 0.957 \\
\hline \multirow[t]{2}{*}{ Watcha scale score } & $\begin{array}{l}\text { Correlation coef- } \\
\text { ficient }\end{array}$ & 0.376 & 0.069 \\
\hline & $P$ value & 0.044 & 0.712 \\
\hline
\end{tabular}

PSQI Pittsburg Sleep Quality Index, PAED Pediatric anesthesia emergence delirium

the school-age group, there were no significant correlations between the preoperative PSQI and PAED (correlation coefficient $=-0.010, p=0.957$ ) or Watcha scale scores (correlation coefficient $=0.069, p=0.712$ ).

\section{Discussion}

Our study demonstrated a correlation between preoperative sleep quality and postoperative ED in pediatric patients who underwent strabismus surgery. Our findings suggest that the poorer preoperative sleep quality and younger age were significantly correlated with the occurrence of ED. Also, in the preschool age group, higher preoperative PSQI scores were associated with higher PAED scale scores. That is, patients with poor preoperative sleep quality showed a higher incidence of ED in preschool age. Nevertheless, the etiology or pathogenesis of ED is unknown, so more research is needed to determine whether the poor preoperative sleep quality is simply accompanied by ED or the cause of ED.

Many factors affect sleep quality. These factors may be biological, psychosocial, or environmental. Biological factors include comorbid medical conditions (e.g., obesity, pain, and asthma), primary sleep disorders (e.g., obstructive sleep apnea and restless legs syndrome), and circadian rhythm changes in adolescents [23]. Psychosocial factors include depression, anxiety, personality disorder, substance abuse, family-related stressors (e.g., parental discord), and academic pressure [17, 20, 23]. Finally, environmental factors include multi-media usage, socioeconomic status, sleep habits, and sleep hygiene [23]. A previous adult-oriented study has shown that a worsening sleep quality was associated with preoperative anxiety [24]. Among the factors that affect sleep quality, obesity, anxiety, pain, personality disorder, and substance abuse are also risk factors for ED [1-4]. Considering these similarities, we hypothesized that there would be a relationship between ED and preoperative sleep quality.
In contrast to the preschool-age group, no association was found between preoperative sleep quality and PAED scale score in the school-age group. There are many factors regarding sleep quality and age can affect those factors itself. Family-related stress, academic pressure, and socioeconomic status have greater influence on sleep quality in the school-age group than in the preschool-age group. Therefore, in the school-age group, environmental factor may be dominant than in preschool-age group.

We enrolled patients between 4 and 12 years of age despite well known risk factor for ED age range is from 2 to 5 years [1]. The PSQI is based on a self-report of conditions and therefore requires proper communication and understanding of the patients. For this reason, children aged 2 to 3 years were ineligible for PSQI interview. Nevertheless, the results of this study were similar to those of previous studies $[1,3,25,26]$. The preschoolage group scored higher on PAED and Watcha scale than the school-age group. Moreover, the preschool-age group had higher incidence of ED than the school-age group (37.9\% vs. 6.7\%). Although the participants' ages in our study differed from those in previous studies, we could demonstrate noticeable age-related changes in the occurrence and severity of ED.

In our study, the overall incidence of ED was $22 \%$, which was lower than that reported in previous studies $[1-3,26]$. First, in our hospital, we allowed parents to stay with the patient before and after anesthesia. As a result, the patient's anxiety level was expected to be relatively low. A child's preoperative anxiety level is another well-known risk factor for ED [5, 27]. The factors related to increase in preoperative anxiety include separation anxiety, anxiety on operation anesthesia, and an unusual environment. Parental presence during anesthetic induction is controversial in reducing anxiety $[28,29]$. Nevertheless, we allowed parents to come along before the induction of anesthesia. In addition, parents were again called to stay beside the patients in the PACU when the surgery was over. One of the parents was even called to stay beside the patient upon arrival at the PACU. Under such circumstances, the patients were more comfortable, less anxious, and cried less compared to when no parents are present in the PACU [30]. The scores of some items on the PAED scale, such as restlessness and inconsolability, might be lower in our study than in previous studies.

Second, adequate pain control with intravenous ketorolac appears to reduce the occurrence of ED. Postoperative pain is a risk factor for ED. We attempted to control postoperative pain with ketorolac $(0.5-1.0 \mathrm{mg} /$ $\mathrm{kg}$, intravenous) in all patients (Table 1). Intravenous ketorolac injection is an effective pain control method in pediatric strabismus surgery [31, 32]. In our study, there was no difference in the dosage of ketorolac according 
to body weight between the two groups. Therefore, it is likely that postoperative pain did not significantly contribute to the differences between the two groups in our study.

Based on our findings, poor preoperative sleep was assumed to be a predictive factor for ED in pediatric patients. If there is a method to improve preoperative sleep quality, then it might be helpful to decrease the incidence of ED. Obstructive sleep apnea is known to cause sleep disruption and has already been reported in studies to be associated with postoperative delirium [12, 14]. Among the PSQI, items D "Cannot breathe comfortably" and E "Cough or snore loudly" of question 5 are questions related to obstructive sleep apnea. In our study, $50 \%$ of patients with a PSQI score of 5 or higher reported snoring in their daily sleep patterns. Several studies have improved sleep quality by correcting sleep hygiene and obstructive sleep apnea [23]. Therefore, further research is needed on the relationship between sleep quality improvement and the occurrence of ED when a modifiable factor of sleep quality is identified and corrected.

This study had some limitations. First, the most important limitation of our study is that a tool for assessing the quality of sleep in children has not been established. Although the PSQI questionnaire is an assessment tool used in previous studies on sleep quality in children, there seem to be some limitations in its application [18, $21,22]$. In the case of younger children, it was difficult to assess the PSQI due to communication problems; thus, children under the age of 4 were excluded from our study. This study obtained a relatively low PSQI $(1.41 \pm 1.65)$ score based on a commonly used cutoff value, which is not suitable for diagnosing sleep disturbance. This result may be because some of the questions in the PSQI questionnaire were not suitable for pediatric patients. PSQI is also not a good parameter for measuring short-term changes in sleep quality. This is because the PSQI questions have been used to assess sleep quality in the past month. The timing of PSQI interview was right after the surgery schedule confirmation. There could be preoperative anxiety which might influence the sleep quality before surgery that is not reflected in our PSQI score. To compensate for this aspect, other sleep quality assessment tools such as polysomnography might be added.

Second, preoperative and postoperative pain assessments were not performed. Pain is an important component of ED; therefore, it would have been better if it had been evaluated. However, intravenous ketorolac injection is effective for pain control in strabismus surgery and has been used routinely in all patients [31, 32]. As there was no difference in the amount of ketorolac used per kilogram of body weight, we assumed that the effect of pain was not significant. Third, in our study, we could not find a significant association between sleep quality and the occurrence of ED in the school-age group. However, since the incidence of ED (6.7\%) was low in school-age group, it seems insufficient to determine the correlation between sleep quality and occurrence of ED. So further studies with more school age patients are needed.

\section{Conclusion}

In conclusion, the incidence of ED tended to be higher in younger age and poorer preoperative sleep quality in pediatric patients. The poorer sleep quality score was associated with higher incidence of ED in the preschoolage. In consideration of the limitations of our study, further studies are required to investigate the correlation between preoperative sleep quality and postoperative ED.

\section{Abbreviations}

BMl: Body mass index; Cl: Confidence interval; ED: Emergence delirium; OR: Odds ratio; PSQI: Pittsburg sleep quality index; PAED: Pediatric anesthesia emergence delirium; PACU: Post-anesthesia recovery unit.

\section{Supplementary Information}

The online version contains supplementary material available at https://doi. org/10.1186/s12871-021-01507-2.

Additional file 1 : Supplementary Table 1. Pediatric anesthesia emergence delirium scale. Supplementary Table 2. Watcha scale.

\section{Acknowledgements \\ Not applicable.}

\section{CONSORT 2010 statement}

The authors have read the CONSORT 2010 statement, and the manuscript was prepared and revised according to the CONSORT 2010 statement.

\section{Authors' contributions}

DW and HJM helped in the study design, planning, conduct, data analysis, and manuscript preparation and revision. $\mathrm{KHS}, \mathrm{KS}$ and $\mathrm{KH}$ helped in the study conduct. LD helped in the study design and manuscript revision. BJ and LHJ helped in the study design, conduct and manuscript revision. All authors have read and approved the manuscript.

\section{Funding}

This work was supported by a 2-Year Research Grant of Pusan National University.

Availability of data and materials

Data are available from the corresponding author on reasonable request.

\section{Declarations}

Ethics approval and consent to participate

The study was approved by the Institutional Review Board of Pusan National University Hospital, Busan, Korea (1704-001-067). Informed consent was obtained from all individual participants included in the study. This study was performed in accordance with the ethical standards of the Declaration of Helsinki (1964) and its subsequent amendments.

Consent for publication

Not applicable. 


\section{Competing interests}

The authors declare they have no competing interests.

\section{Author details}

1 Biomedical Research Institute, Pusan National University Hospital, Busan 49241, Republic of Korea. ${ }^{2}$ Department of Anesthesia and Pain Medicine, School of Medicine, Pusan National University, 1-10, Ami-dong, Seo-gu, Busan 49241, Republic of Korea.

Received: 28 June 2021 Accepted: 8 November 2021

Published online: 22 November 2021

\section{References}

1. Vlajkovic GP, Sindjelic RP. Emergence delirium in children: many questions, few answers. Anesth Analg. 2007;104(1):84-91.

2. Silva $L M, B r a z L G, M o ́ d o l o ~ N S$. Emergence agitation in pediatric anesthesia: current features. J Pediatr. 2008;84(2):107-13.

3. Lee SJ, Sung TY. Emergence agitation: current knowledge and unresolved questions. Korean J Anesthesiol. 2020;73(6):471-85.

4. Lepousé C, Lautner CA, Liu L, Gomis P, Leon A. Emergence delirium in adults in the post-anaesthesia care unit. Br J Anaesth. 2006;96(6):747-53.

5. Abu-Shahwan I. Effect of propofol on emergence behavior in children after sevoflurane general anesthesia. Paediatr Anaesth. 2008;18(1):55-9.

6. Kawai M, Kurata S, Sanuki T, Mishima G, Kiriishi K, Watanabe T, et al. The effect of midazolam administration for the prevention of emergence agitation in pediatric patients with extreme fear and non-cooperation undergoing dental treatment under sevoflurane anesthesia, a doubleblind, randomized study. Drug Des Devel Ther. 2019;13:1729-37.

7. Demir CY, Yuzkat N. Prevention of emergence agitation with ketamine in rhinoplasty. Aesthet Plast Surg. 2018;42(3):847-53.

8. Sun Y, Li Y, Sun Y, Wang $X, Y$, H, Yuan X. Dexmedetomidine effect on emergence agitation and delirium in children undergoing laparoscopic hernia repair: a preliminary study. J Int Med Res. 2017:45(3):973-83.

9. Luo ZY, Li LL, Wang D, Wang HY, Pei FX, Zhou ZK. Preoperative sleep quality affects postoperative pain and function after total joint arthroplasty: a prospective cohort study. J Orthop Surg Res. 2019;14(1):378.

10. Yang MMH, Hartley RL, Leung AA, Ronksley PE, Jetté N, Casha S, et al. Preoperative predictors of poor acute postoperative pain control: a systematic review and meta-analysis. BMJ Open. 2019;9(4):e025091.

11. Wang JP, Lu SF, Guo LN, Ren CG, Zhang ZW. Poor preoperative sleep quality is a risk factor for severe postoperative pain after breast cancer surgery: a prospective cohort study. Medicine (Baltimore) 2019;98(44):e17708.

12. Lam EWK, Chung F, Wong J. Sleep-disordered breathing, postoperative delirium, and cognitive impairment. Anesth Analg. 2017;124(5):1626-35.

13. Leung JM, Sands LP, Newman S, Meckler G, Xie Y, Gay C, et al. Preoperative sleep disruption and postoperative delirium. J Clin Sleep Med. 2015;11(8):907-13.

14. Roggenbach J, Klamann M, von Haken R, Bruckner T, Karck M, Hofer S. Sleep-disordered breathing is a risk factor for delirium after cardiac surgery: a prospective cohort study. Crit Care. 2014;18(5):477.

15. Todd OM, Gelrich L, MacLullich AM, Driessen M, Thomas C, Kreisel SH. Sleep disruption at home as an independent risk factor for postoperative delirium. J Am Geriatr Soc. 2017;65(5):949-57.
16. Forbes EE, Bertocci MA, Gregory AM, Ryan ND, Axelson DA, Birmaher B, et al. Objective sleep in pediatric anxiety disorders and major depressive disorder. J Am Acad Child Adolesc Psychiatry. 2008;47(2):148-55.

17. Micic G, Lovato N, Gradisar M, Lack LC. Personality differences in patients with delayed sleep-wake phase disorder and non-24-h sleep-wake rhythm disorder relative to healthy sleepers. Sleep Med. 2017;30:128-35.

18. Miniksar DY, Özdemir M. Sleep quality in children and adolescents with obsessive-compulsive disorders. Nord J Psychiatry. 2021;75(1):25-30.

19. Sannar EM, Palka T, Beresford C, Peura C, Kaplan D, Verdi M, et al. Sleep problems and their relationship to maladaptive behavior severity in psychiatrically hospitalized children with autism spectrum disorder (ASD). J Autism Dev Disord. 2018;48(11):3720-6.

20. Van Veen MM, Karsten J, Lancel M. Poor sleep and its relation to impulsivity in patients with antisocial or borderline personality disorders. Behav Med. 2017;43(3):218-26.

21. Gozmen S, Keskin S, Akil I. Enuresis nocturna and sleep quality. Pediatr Nephrol. 2008;23(8):1293-6.

22. Ayaki M, Torii H, Tsubota K, Negishi K. Decreased sleep quality in high myopia children. Sci Rep. 2016;6:33902.

23. Moturi S, Avis K. Assessment and treatment of common pediatric sleep disorders. Psychiatry (Edgmont). 2010;7(6):24-37.

24. Wright CE, Schnur JB, Montgomery GH, Bovbjerg DH. Psychological factors associated with poor sleep prior to breast surgery: an exploratory study. Behav Med. 2010;36(3):85-91.

25. Bong CL, Ng AS. Evaluation of emergence delirium in Asian children using the pediatric anesthesia emergence delirium scale. Paediatr Anaesth. 2009;19(6):593-600.

26. Mason KP. Paediatric emergence delirium: a comprehensive review and interpretation of the literature. Br J Anaesth. 2017;118(3):335-43.

27. Stargatt R, Davidson AJ, Huang GH, Czarnecki C, Gibson MA, Stewart $\mathrm{SA}$, et al. A cohort study of the incidence and risk factors for negative behavior changes in children after general anesthesia. Paediatr Anaesth. 2006;16(8):846-59.

28. Sadeghi A, Khaleghnejad Tabari A, Mahdavi A, Salarian S, Razavi SS. Impact of parental presence during induction of anesthesia on anxiety level among pediatric patients and their parents: a randomized clinical trial. Neuropsychiatr Dis Treat. 2017;12:3237-41.

29. Chundamala J, Wright JG, Kemp SM. An evidence-based review of parental presence during anesthesia induction and parent/child anxiety. Can J Anaesth. 2009;56(1):57-70.

30. Fina DK, Lopas LJ, Stagnone JH, Santucci PR. Parent participation in the postanesthesia care unit: fourteen years of progress at one hospital. J Perianesth Nurs. 1997;12(3):152-62.

31. Mendel HG, Guarnieri KM, Sundt LM, Torjman MC. The effects of ketorolac and fentanyl on postoperative vomiting and analgesic requirements in children undergoing strabismus surgery. Anesth Analg. 1995;80(6):1129-33.

32. Munro HM, Riegger LQ, Reynolds PI, Wilton NC, Lewis $\mathbf{H}$. Comparison of the analgesic and emetic properties of ketorolac and morphine for paediatric outpatient strabismus surgery. Br J Anaesth. 1994;72(6):624-8.

\section{Publisher's Note}

Springer Nature remains neutral with regard to jurisdictional claims in published maps and institutional affiliations. 